\title{
TS gene polymorphisms are not good markers of response to 5-FU therapy in stage III colon cancer patients
}

\author{
A. Fariña-Sarasqueta ${ }^{\text {a,b }}$, M.J.E.M. Gosens ${ }^{\text {b }}$, E. Moerland ${ }^{\text {a }}$, I. van Lijnschoten ${ }^{\text {a }}$, V.E.P.P. Lemmens ${ }^{\text {c }}$, \\ G.D. Slooter ${ }^{\mathrm{d}}$, H.J.T. Rutten ${ }^{\mathrm{e}}$ and A.J.C. van den Brule ${ }^{\mathrm{a}, \mathrm{b}, \mathrm{f}, *}$ \\ a PAMM Laboratory for Pathology, Eindhoven, The Netherlands \\ ${ }^{\mathrm{b}}$ Fontys University of Applied Science, Centre for Medical Molecular Diagnostics, Eindhoven, The Netherlands \\ ${ }^{\mathrm{c}}$ Comprehensive Cancer Centre South, Eindhoven, The Netherlands \\ d Department of Surgery, Máxima Medical Centre Veldhoven, Veldhoven, The Netherlands \\ e Department of Surgical Oncology, Catharina Hospital Eindhoven, Eindhoven, The Netherlands \\ ${ }^{\mathrm{f}}$ Department of Molecular Diagnostics, Catharina Hospital Eindhoven, Eindhoven, The Netherlands
}

\begin{abstract}
Aim: Although the predictive and prognostic value of thymidylate synthase (TS) expression and gene polymorphism in colon cancer has been widely studied, the results are inconclusive probably because of methodological differences. With this study, we aimed to elucidate the role of TS gene polymorphisms genotyping in therapy response in stage III colon carcinoma patients treated with 5-FU adjuvant chemotherapy.

Patients and methods: 251 patients diagnosed with stage III colon carcinoma treated with surgery followed by 5-FU based adjuvant therapy were selected. The variable number of tandem repeats (VNTR) and the single nucleotide polymorphism (SNP) in the $5^{\prime}$-untranslated region of the TS gene were genotyped.

Results: There was a positive association between tumor T stage and the VNTR genotypes $(p=0.05)$.

In both univariate and multivariate survival analysis no effects of the studied polymorphisms on survival were found. However, there was an association between both polymorphisms and age. Among patients younger than 60 years, the patients homozygous for $2 \mathrm{R}$ seemed to have a better overall survival, whereas among the patients older than 67 this longer survival was seen by the carriers of other genotypes.

Conclusion: We conclude that the TS VNTR and SNP do not predict response to 5-FU therapy in patients with stage III colon carcinoma. However, age appears to modify the effects of TS polymorphisms on survival.
\end{abstract}

Keywords: Colon carcinoma, TS, VNTR, SNP, survival, 5-FU, age

\section{Introduction}

5-Fluorouracil (5-FU) is the chemotherapeutic drug of choice in the treatment of colon cancer. 5-FU causes cell death through two different mechanisms [28]. One mechanism is incorporation of fluorouracil triphosphate (FUTP) into RNA causing disruption of normal RNA processes. The second mechanism of action consists on inhibition of thymidylate synthase (TS). TS

\footnotetext{
*Corresponding author: Adriaan J.C. van den Brule, PhD, Department of Molecular Diagnostics, PAMM Laboratory for Pathology and Catharina Hospital Eindhoven, Michelangelolaan 2, 5623 EJ, Eindhoven, The Netherlands. Tel.: +31 4023961 00; Fax: +31 40 23961 09; E-mail: a.van.den.brule@pamm.nl.
}

provides the sole de novo source of thymidylate for DNA synthesis, thus TS inhibition causes depletion of nucleotides disrupting DNA synthesis and repair. Besides, it also causes DNA damage through misincorporation of deoxyuracil triphosphate (dUTP) into the DNA strand [28]. The fact that enhanced TS protein expression has been described as a mechanism of acquired 5-FU resistance [37] supports the thesis that TS inhibition is the main mechanism of action of 5-FU.

Because of its role as potential main target of 5-FU, TS has been widely studied as a molecular maker of therapy response in colorectal cancer, without conclusive results. Several studies have focussed on quantitation of TS protein by immunohistochemistry (IHC) 


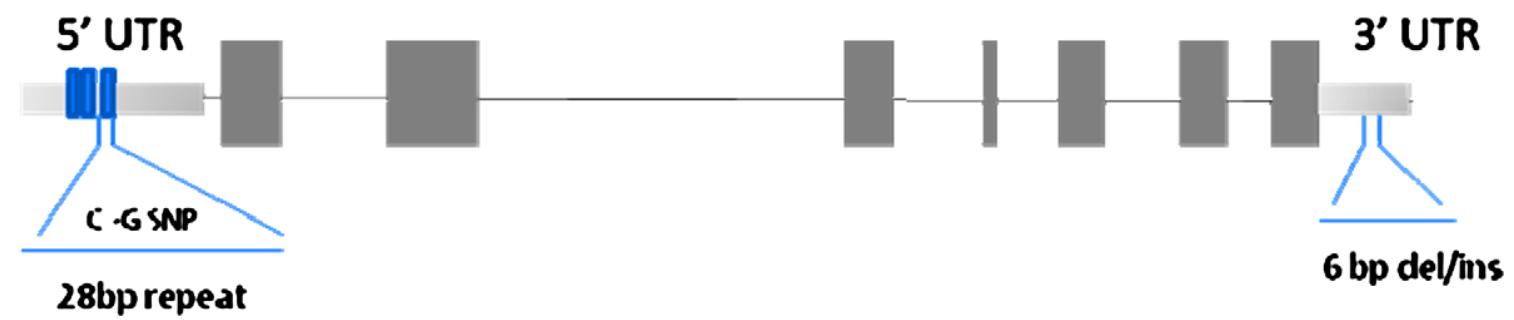

Fig. 1. Schematic representation of the TYMS gene with known polymorphisms in $5^{\prime}$ untranslated region $\left(5^{\prime}\right.$-UTR) and $3^{\prime}$-UTR. On the $5^{\prime}$-UTR the 28 bp repeat with the SNP in the third repeat. On the $3^{\prime}$-UTR a 6 bp long deletion/insertion.

$[3,6,8,9,18,23,25,39,48,51]$ or mRNA expression $[1$, $2,16,23,27,33,43,44,49,52,53]$ in tumors and metastasis whereas others have focussed on gene polymorphisms genotyping $[4,5,7,9,13,15,17,20-22,24,32,41$, 42,45-48,50]. Besides technical differences, heterogeneity in patient selection also plays a role in the lack of consistency between results. Many studies for instance have included patients with rectal cancer [13,24, $32,47]$, while these are treated differently than colon cancer. Furthermore, some reports described heterogeneous cohorts of patients including all disease stages and patients who did not receive 5 -FU based adjuvant therapy at all $[5,13,24,46,47]$. Results are therefore frequently contradictory [38].

We have recently reported the reliability of different methods for TS typing, like genotyping of three known gene polymorphisms (see Fig. 1), TS protein expression quantitation, TS gene amplification and loss of heterozygosity in predicting 5-FU therapy response [12]. From these results, it seemed that genotyping of the $5^{\prime}$-untraslated region polymorphism of the TS gene was more reliable for predicting response to therapy than protein expression, as determined by IHC and than genotyping the rest of polymorphisms in the $3^{\prime}$ UTR.

The aim of this study was to determine the value of the TS gene $5^{\prime}$-UTR polymorphisms as a possible molecular marker for 5-FU response in a well defined, homogeneous population of stage III colon cancer patients who had been treated with 5-FU based adjuvant chemotherapy.

\section{Materials and methods}

\subsection{Patients}

All patients $(n=251)$ were stage III colon carcinoma patients treated with surgery followed by $5-\mathrm{FU}$ based adjuvant chemotherapy between 1995 and 2004 in four different hospitals in the Eindhoven area in the south of The Netherlands.

Two hundred forty two patients $(96.4 \%)$ received 5FU in combination with levamisol following the Mayo regime, 4 patients $(1.6 \%)$ had 5 -FU plus leucovorin and finally 5 patients $(2 \%)$ received capecitabine.

Routine histopathological diagnoses were performed in a central laboratory, the PAMM laboratory for Pathology in Eindhoven. Epidemiological data and tumor characteristics of all patients included were extracted from the Eindhoven Cancer Registry of the Comprehensive Cancer Centre South (IKZ, The Netherlands). Follow up information was obtained from the medical records of these patients. The research protocol was approved by the Scientific Committee of the Catharina Hospital Eindhoven.

\subsection{VNTR typing}

DNA was obtained after proteinase $\mathrm{K}$ digestion of 5 sections of $5 \mu \mathrm{m}$ from formalin fixed paraffin embedded (FFPE) blocks with normal colonic tissue. Subsequently, the tissue digest was purified with HPPTP purification kit for genomic DNA (Roche diagnostics, Almere, The Netherlands). PCR for the VNTR was performed using the following primers: (forward) $5^{\prime}$ gcg gaa ggg gtc ctg cca- $3^{\prime}$ and (reverse) $5^{\prime}$-tcc gag ccg gcc aca ggc at- $3^{\prime}$. The reaction was performed in $50 \mu \mathrm{l}$ final volume as described elsewhere [19]. PCR products were separated by electrophoresis on a $2 \%$ agarose gel. The expected product sizes were $107 \mathrm{bp}$ for the $2 \mathrm{R}$ allele and $135 \mathrm{bp}$ for the $3 \mathrm{R}$ allele.

\subsection{SNP genotyping}

Subsequently, the previously obtained PCR products were digested by HaeIII restriction enzyme during one hour at $37^{\circ} \mathrm{C}$ (New England Biolabs, Ipswich, UK). The $\mathrm{G}$ to $\mathrm{C}$ base change removes a HaeIII restriction 
site present at position 12 of the second $28 \mathrm{bp}$ repeat of the $3 R$ allele. PCR products of carriers of the $\mathrm{G}$ allele will be digested giving an additional shorter band of 66 bp after gel electrophoresis on a 3\% agarose gel.

\subsection{Statistical analysis}

Statistical analyses were performed using SPSS software package for Windows (Chicago, IL, USA). Categorical data were analyzed by means of a chisquare or Fischer's exact test. To study the difference in median age between the different VNTR and SNP genotype groups, age was used as a continuous variable to perform a Kruskal-Wallis test. After this, age at diagnosis was categorized according to tertiles for further analyses.

To study the effects of the different polymorphisms on 5-FU response, survival analysis was used.

The univariate survival analysis was performed using the Kaplan-Meier test. Differences between survival curves were tested for significance by the Logrank test. Overall survival was the time between surgery and death discriminating between death because of colon cancer or because of other reasons when this was specified in the medical records. Disease free survival was the time between surgery and disease progression. Disease specific survival was defined as the time between surgery and death because of colon cancer. Cox proportional hazards regression analysis was used for multivariate survival analyses. All tests were two-tailed and $p<0.05$ was considered to be statistically significant.

\section{Results}

\subsection{Clinicopathological characteristics}

Patient and tumor histopathological characteristics are shown in Table 1. All patients had positive lymph nodes and no recognizable distant metastasis at time of diagnosis. 10 patients (4\%) developed distant metastasis within the first four months following surgery.

Median follow-up was 47 months (range 2133 months). 122 patients (49\%) were still alive at the end of the follow up period, 30 patients $(12 \%)$ were alive but had disease progression, $80(32 \%)$ died due to cancer related causes and 17 patients $(7 \%)$ died due to non cancer related causes according to the medical records. Finally, medical records of two patients were incomplete and their follow-up status was unknown.

\subsection{VNTR distribution}

VNTR distribution and association with studied variables is shown in Table 1. Distribution of the VNTR in the population studied followed Hardy Weinberg equilibrium. There was a significant association between tumor T stage and VNTR alleles. Patients homozygous for the $2 \mathrm{R}$ allele had significantly more frequently low $\mathrm{T}$ stages than did heterozygous and homozygous $3 \mathrm{R}(p=0.05)$.

There was, further, a significant association between age at diagnosis and the three genotypes. Median age in the group with the $3 \mathrm{R} / 3 \mathrm{R}$ genotype was significantly lower than median age in the $2 R / 2 R$ and in the 2R/3R group; 61 years vs. 64 and 65 , respectively ( $H=14.633, p=0.001,99 \%$ CI $0.000-0.001)$. To further study the association between age at diagnosis and genotypes and their role in survival, we categorize age in three different groups according to tertiles. These tertile groups corresponded in our study population to the following age categories; younger than 60 years, between 60-67 years, and older than 67 years, respectively. There was a significant relationship between the three genotypes and the three age categories $(p=0.02)$.

\subsection{SNP distribution}

Two hundred and thirteen out of 251 patients had enough PCR product available to study the $\mathrm{G}>\mathrm{C}$ SNP present in the second repeat of the $3 \mathrm{R}$ allele.

Frequencies of the different SNP alleles in our patient population were in agreement with the in the literature published frequencies and are shown in Table 2. There was no significant association between the different SNP alleles and any of the categorical variables tested.

Age was tested as a continuous variable and there was a significant association with the SNP genotypes ( $H=15.135, p=0.01,99 \%$ CI 0.006-0.01). Median age in the $3 \mathrm{G} / 3 \mathrm{C}$ group was 53,5 years, whereas all the other genotype groups had a median age greater than 60 years (Fig. 2). When age was categorized according to tertiles, a positive trend was seen towards an association between age tertiles and the $\operatorname{SNP}(p=0.06)$. 
Table 1

Patient's characteristics. Histopathological features of the tumors in relation to VNTR distribution

\begin{tabular}{|c|c|c|c|c|c|}
\hline \multirow[t]{2}{*}{ Demographic and histopathological characteristics } & \multicolumn{4}{|c|}{$n(\%)$} & \multirow[t]{2}{*}{$p$} \\
\hline & Total & $\begin{array}{l}2 \mathrm{R} / 2 \mathrm{R} \\
59(23)\end{array}$ & $\begin{array}{c}2 \mathrm{R} / 3 \mathrm{R} \\
128(51)\end{array}$ & $\begin{array}{l}3 R / 3 R \\
64(26)\end{array}$ & \\
\hline \multicolumn{6}{|l|}{ Gender } \\
\hline Female & $112(45)$ & $23(40)$ & $58(45)$ & $31(48)$ & 0.6 \\
\hline Male & $139(55)$ & $35(60)$ & $70(55)$ & $33(52)$ & \\
\hline \multicolumn{6}{|l|}{ Age } \\
\hline First tertile $\leqslant 59$ & $78(31)$ & $17(29)$ & $31(24)$ & $30(47)$ & 0.02 \\
\hline Second tertile $60-67$ & $88(35)$ & $21(36)$ & $46(36)$ & $21(33)$ & \\
\hline Third tertile $>67$ & $85(34)$ & $20(35)$ & $51(40)$ & $13(20)$ & \\
\hline Median age & 64 & 64 & 5 & 61 & \\
\hline \multicolumn{6}{|l|}{ Tumor location } \\
\hline Right & $133(54)$ & $29(50)$ & $73(57.5)$ & $31(51)$ & 0.5 \\
\hline Left & $114(46)$ & $29(50)$ & $54(42.5)$ & $30(49)$ & \\
\hline \multicolumn{6}{|l|}{ T stage } \\
\hline $\mathrm{T}_{1}$ & $1(0.4)$ & $1(2)$ & $0(0)$ & $0(0)$ & 0.05 \\
\hline $\mathrm{T}_{2}$ & $22(8.6)$ & $9(15)$ & $9(7)$ & $4(6)$ & \\
\hline $\mathrm{T}_{3}$ & $183(73)$ & $44(76)$ & $94(73)$ & $45(70)$ & \\
\hline $\mathrm{T}_{4}$ & $45(18)$ & $4(7)$ & $25(20)$ & $15(24)$ & \\
\hline \multicolumn{6}{|l|}{ Positive lymph nodes } \\
\hline $1-3 \mathrm{~N}_{1}$ & $135(70)$ & $32(70)$ & $67(70.5)$ & $36(69)$ & 0.9 \\
\hline$\geqslant 4 \mathrm{~N}_{2}$ & $58(30)$ & $14(30)$ & $28(29.5)$ & $16(31)$ & \\
\hline \multicolumn{6}{|l|}{ Differentiation grade } \\
\hline Well-differentiated & $28(12)$ & $6(11)$ & $16(13)$ & $6(10)$ & 0.6 \\
\hline Moderated & $148(61.6)$ & $36(65)$ & $69(58)$ & $39(64)$ & \\
\hline Poor & $62(26)$ & $12(22)$ & $34(29)$ & $16(26)$ & \\
\hline Undifferentiated & $1(0.4)$ & $1(2)$ & $0(0)$ & $0(0)$ & \\
\hline
\end{tabular}

\subsection{Categorization into high and low TS expression}

Based on the effects of the VNTR in TS protein expression as described in the literature, our patient population was divided in two putative categories low and high TS expression, according to the genotypes found: homozygous $2 R$ and carriers of the $3 R$ allele $(3 R / 3 R$, $2 \mathrm{R} / 3 \mathrm{R}$ ), respectively $[19,20,22,31,41]$.

When additionally the SNP genotypes were included, patients can be divided in the following groups: putative high TS expression as carriers of the $\mathrm{G}$ allele (3RG/3RG, 3RG/3RC, 2R/3RG) and putative low TS expression as carriers of the $\mathrm{C}$ allele plus the $2 \mathrm{R}$ homozygous ( $2 R / 2 R, 2 R / 3 R C, 3 R C / 3 R C) .60 \%$ of the patients were categorized as putative low expression vs. $40 \%$ putative high expression.

\subsection{Survival analysis}

Analysis of the total population revealed no associations between the genotypes, either independently or in categories (as low and high expression), and overall survival, disease free survival or cancer specific survival (Fig. 3A and B). These results were confirmed by a multivariable Cox proportional hazard model including the following variables; $\mathrm{T}$ stage, $\mathrm{N}$ stage, differentiation grade, sex, tumor location and TS SNP category or VNTR category.

Mean time to progression in the groups according to SNP and VNTR category did not differ significantly (SNP category low 42 months and high 44 months. VNTR category low 42 months and high 42 months). 
Table 2

Distribution of SNP genotypes according to histopathological features

\begin{tabular}{|c|c|c|c|c|c|c|c|}
\hline \multirow[t]{4}{*}{ Demographic and histopathological characteristics } & \multicolumn{6}{|c|}{$n(\%)$} & \multirow[t]{4}{*}{$p$} \\
\hline & \multirow[t]{3}{*}{ 2R/2R 59 (28) } & \multicolumn{2}{|c|}{$2 \mathrm{R} / 3 \mathrm{R}$} & \multicolumn{3}{|c|}{$3 \mathrm{R} / 3 \mathrm{R}$} & \\
\hline & & $2 \mathrm{R} / 3 \mathrm{RC}$ & $2 \mathrm{R} / 3 \mathrm{RG}$ & $3 R C / 3 R G$ & $3 \mathrm{RC} / 3 \mathrm{RC}$ & $3 R G / 3 R G$ & \\
\hline & & $50(23)$ & $53(25)$ & $14(7)$ & $20(9)$ & $17(8)$ & \\
\hline \multicolumn{8}{|l|}{ Gender } \\
\hline Female & $24(41)$ & $24(48)$ & $21(40)$ & $6(43)$ & $14(70)$ & $7(41)$ & 0.3 \\
\hline Male & $35(59)$ & $26(52)$ & $32(60)$ & $8(57)$ & $6(30)$ & $10(59)$ & \\
\hline \multicolumn{8}{|l|}{ Age } \\
\hline First tertile $\leqslant 59$ & $17(29)$ & $12(24)$ & $11(21)$ & $10(72)$ & $5(25)$ & $7(42)$ & 0.06 \\
\hline Second tertile $60-67$ & $21(36)$ & $21(42)$ & $20(38)$ & $2(14)$ & $10(50)$ & $5(29)$ & \\
\hline Third tertile $>67$ & $21(36)$ & $17(34)$ & $22(41)$ & $2(14)$ & $5(25)$ & $5(39)$ & \\
\hline Median age & 64 & 65 & 66 & 64 & 53.5 & 65 & \\
\hline \multicolumn{8}{|l|}{ Tumor Location } \\
\hline Right & $29(49)$ & $25(50)$ & $35(67)$ & $8(57)$ & $9(47)$ & $10(62.5)$ & 0.4 \\
\hline Left & $30(51)$ & $25(50)$ & $17(33)$ & $6(43)$ & $10(53)$ & $6(37.5)$ & \\
\hline \multicolumn{8}{|l|}{ T stage } \\
\hline $\mathrm{T}_{1}$ & $1(2)$ & $0(0)$ & $0(0)$ & $0(0)$ & $0(0)$ & $0(0)$ & 0.2 \\
\hline $\mathrm{T}_{2}$ & $9(15)$ & $3(6)$ & $4(7.5)$ & $0(0)$ & $1(5)$ & $3(18)$ & \\
\hline $\mathrm{T}_{3}$ & $45(76)$ & $35(70)$ & $40(75.5)$ & $11(79)$ & $15(75)$ & $8(47)$ & \\
\hline $\mathrm{T}_{4}$ & $4(7)$ & $12(24)$ & $9(17)$ & $3(21)$ & $4(20)$ & $6(35)$ & \\
\hline \multicolumn{8}{|l|}{$N$ stage } \\
\hline $\mathrm{N}_{1}$ & $33(70)$ & $28(72)$ & $22(61)$ & $9(75)$ & $11(79)$ & $8(50)$ & 0.5 \\
\hline $\mathrm{N}_{2}$ & $14(30)$ & $11(28)$ & $14(39)$ & $3(25)$ & $3(21)$ & $8(50)$ & \\
\hline \multicolumn{8}{|l|}{ Differentiation grade } \\
\hline Well-differentiated & $6(11)$ & $4(9)$ & $7(14)$ & $1(8)$ & $4(20)$ & $1(6)$ & 0.7 \\
\hline Moderated & $37(66)$ & $27(59)$ & $31(63)$ & $10(83)$ & $12(60)$ & $9(53)$ & \\
\hline Poor & $12(21)$ & $15(33)$ & $11(23)$ & $1(8)$ & $4(20)$ & $7(41)$ & \\
\hline Undifferentiated & $1(2)$ & $0(0)$ & $0(0)$ & $0(0)$ & $0(0)$ & $0(0)$ & \\
\hline
\end{tabular}

Since there was an association between TS gene polymorphisms and age, we stratified to age tertiles to study the effect of the polymorphism on survival in relation to age. As shown in Fig. 4, there was a difference between old and young patients. Moreover, this difference could be seen when we classified the patients as putative low and putative high TS expression according to the SNP (Fig. 4A) and to the VNTR alone (Fig. 4B). There was a switch in the genotype associated to longer overall survival as the patients age increased. In other words, among patients younger than 60 years, the $2 \mathrm{R}$ homozygous had a better overall survival $(p=0.02)$ whereas between patients older than 67 years, the ones with putative high TS expression ( $\mathrm{G}$ allele) had a longer overall survival $(p=0.06)$. These age dependent relations were also seen for CSS albeit not significant. However, there was no age dependent effect for DFS.

\section{Discussion}

Although, several studies have been published about the value of TS in colorectal cancers, as reviewed by Popat [38], the results are often contradictory and inconclusive particularly in patients treated adjuvantly.

Therefore this study aimed to elucidate the value of TS gene polymorphisms as possible molecular marker of therapy response in stage III colon carcinoma patients treated with adjuvant 5-FU chemotherapy.

In our well defined population of stage III colon cancer patients, TS genotype as determined by the SNP 


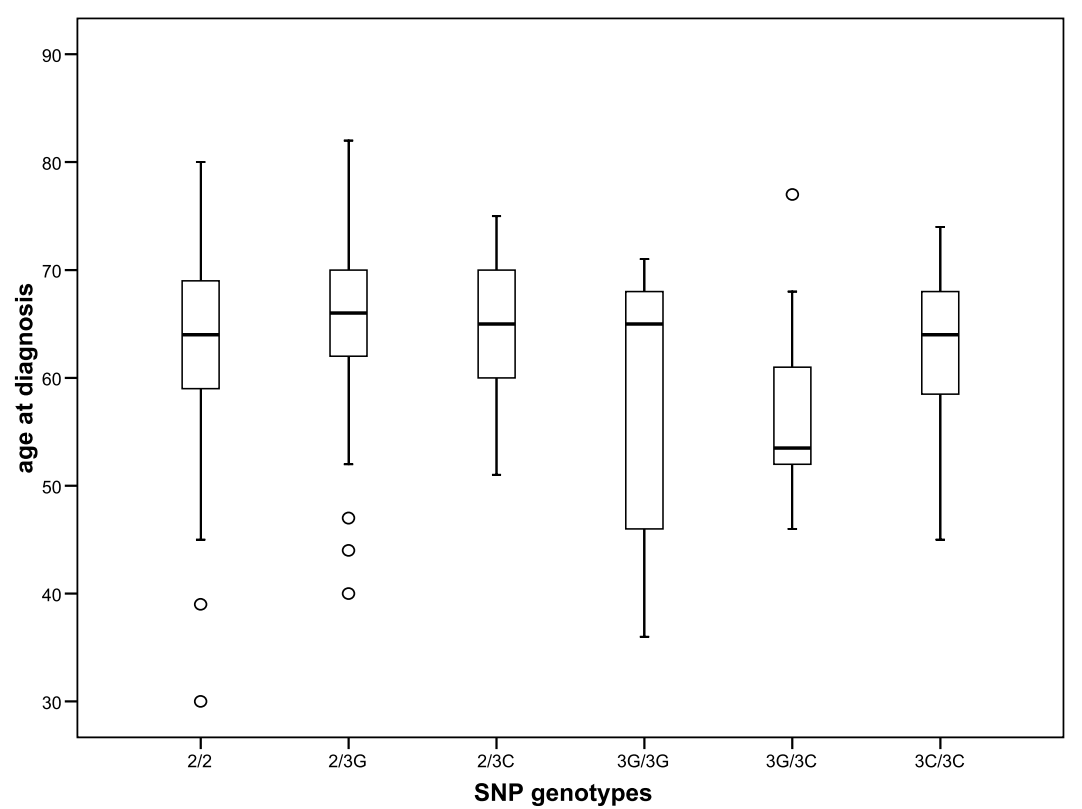

Fig. 2. Age distribution according to SNP genotypes (Kruskal-Wallis $H=15.135, p=0.01,99 \%$ CI 0.006-0.0.

and the VNTR on the $5^{\prime}$-UTR of the gene had no effect on patient outcome. There were no differences in survival (OS, DFS, CSS) between patients according to the genotypes independently or categorized as high en low TS expression based on either the TS SNP or on the VNTR alone (Fig. 3A and B). Although, in a previous publication we reported a predictive value for the VNTR [12], only a small number of patients were studied at that time and the apparently contradictory results could be explained by the difference in patient numbers between studies. In the present larger cohort of patients, we were not able to reproduce our previous results.

Moreover, inconsistent results over the predictive value of TS genotype and phenotype are a common feature in the literature. To our knowledge, there are at least, seven reports studying the value of both $5^{\prime}$-UTR polymorphisms, VNTR and SNP, in colorectal carcinoma $[9,22,24,29,32,40,42]$. Our study agrees with Lecomte et al., Ruzzo et al. and Prall et al.; partly with Fernandez Contreras et al. and argues with Kawakami et al., Marcuello et al. and Lurje et al. In contrast with our patient population which consisted in stage III colon carcinoma patients only, all the previous publications included rectal carcinomas and studied either advanced colorectal cancer $[32,42]$ or combined different disease stages [9,24,29]. We excluded rectal cancer patients because their treatment differs greatly from that of colon cancer patients. Rectal and colon cancer are likely to be two different diseases arising from different pathogenetic pathways and with different clinical behaviours [10]. We, as Prall et al. included only stage III patients in order to have a homogeneous population. Accordingly, similar results were found although Prall and co workers included rectal cancer patients, albeit not neoadjuvantly treated and their patients' population was smaller [40].

Several authors have described a functional role of the TS 5'-UTR polymorphisms on TS protein expression. 3R allele and $G$ allele carriers would have a higher TS protein level than homozygous $2 \mathrm{R} / 2 \mathrm{R}$ or $\mathrm{C}$ allele carriers [19,20,22,31,41]. A higher TS expression has been described as a mechanism of 5-FU resistance [37], hence one would expect that $3 \mathrm{R}$ allele and $\mathrm{G}$ allele carriers would respond worse to 5-FU and have a poorer survival. Our results do not support this thesis. However, the regulation of TS expression and function remains quite complex and most likely is influenced by many still unknown factors [11, 37]. Thus, ideally to explain the biological role of TS in the resistance to 5-FU, other techniques to objectively study protein expression and preferably function, would probably be more accurate. Therefore, our results based on DNA genotyping should not be interpreted as a biological explanation of 5-FU resistance mechanisms but as an answer to whether genotyping is a good marker for therapy response in colon cancer patients. 

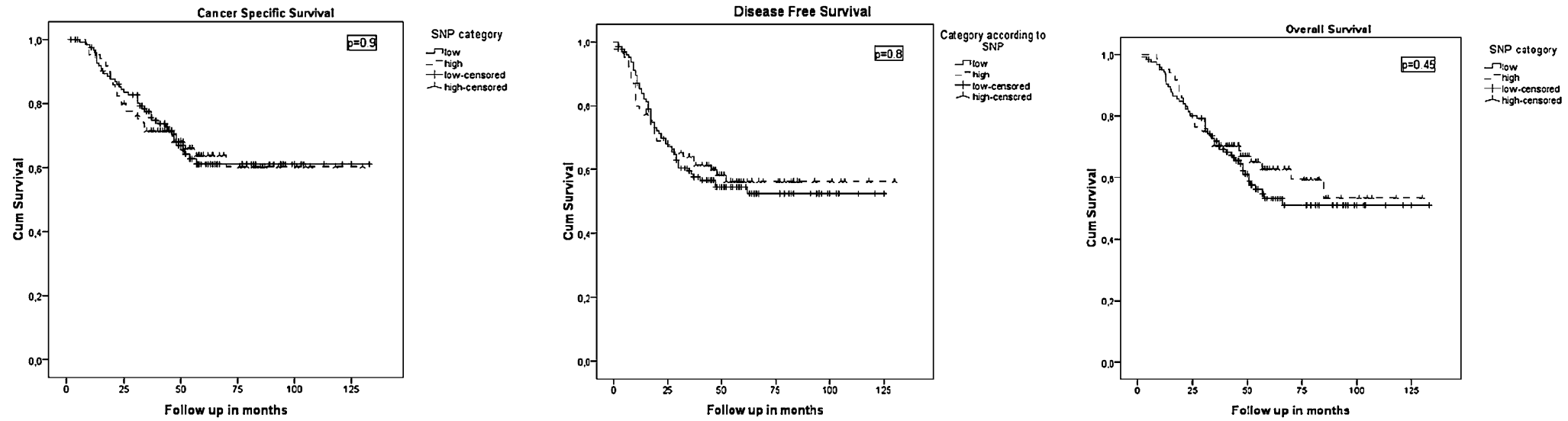

(A)
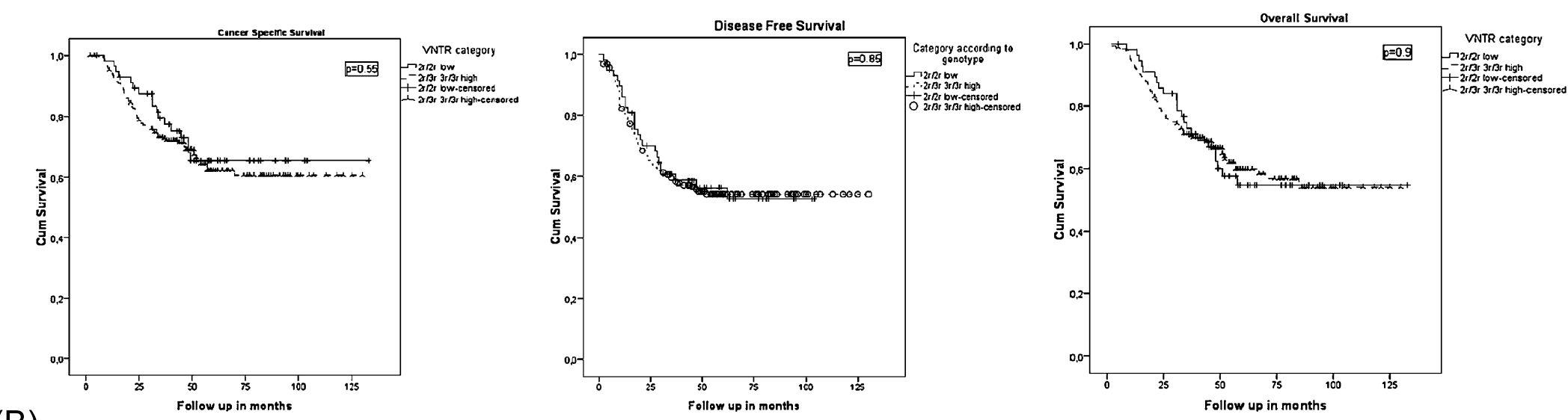

Fig. 3. Kaplan-Meier schemas for OS, DFS and CSS according to (A) SNP categories TS low (2R homozygous plus C allele) and high (G allele) producers (B) VNTR categories (2R/2R low vs. $2 \mathrm{R} / 3 \mathrm{R}$ and $3 \mathrm{R} / 3 \mathrm{R}$ high). 
Overall Survive

Age First tertile $<60$ years

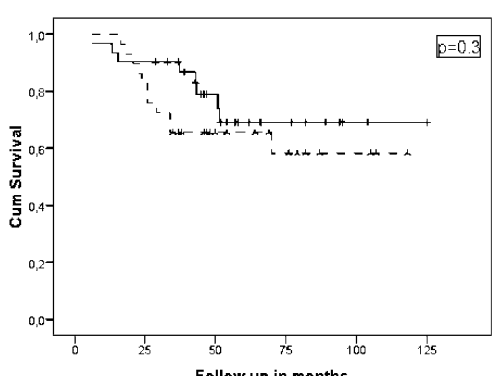

(A)

Overall Survival

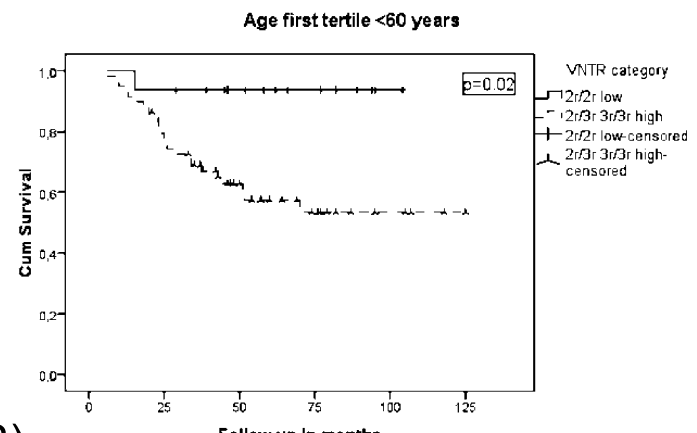

Overall Survival

Age Second Tertile 60-67 years

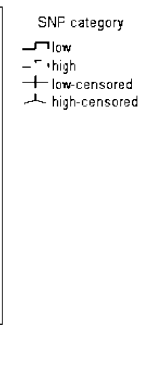

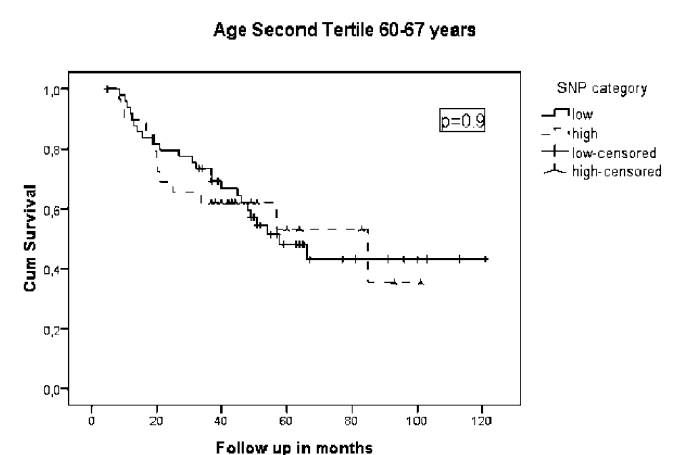

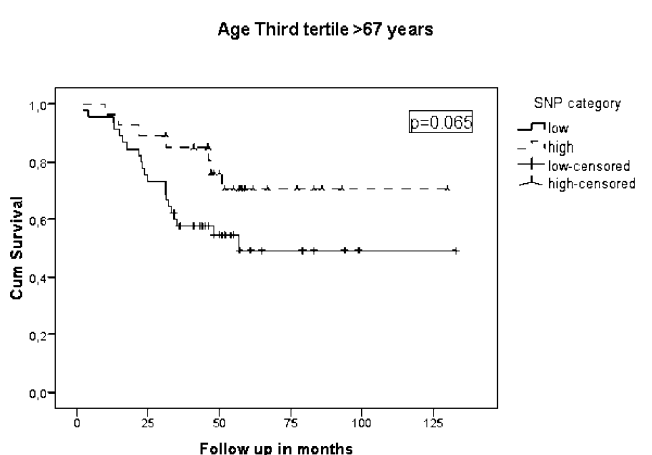

Overall Survival
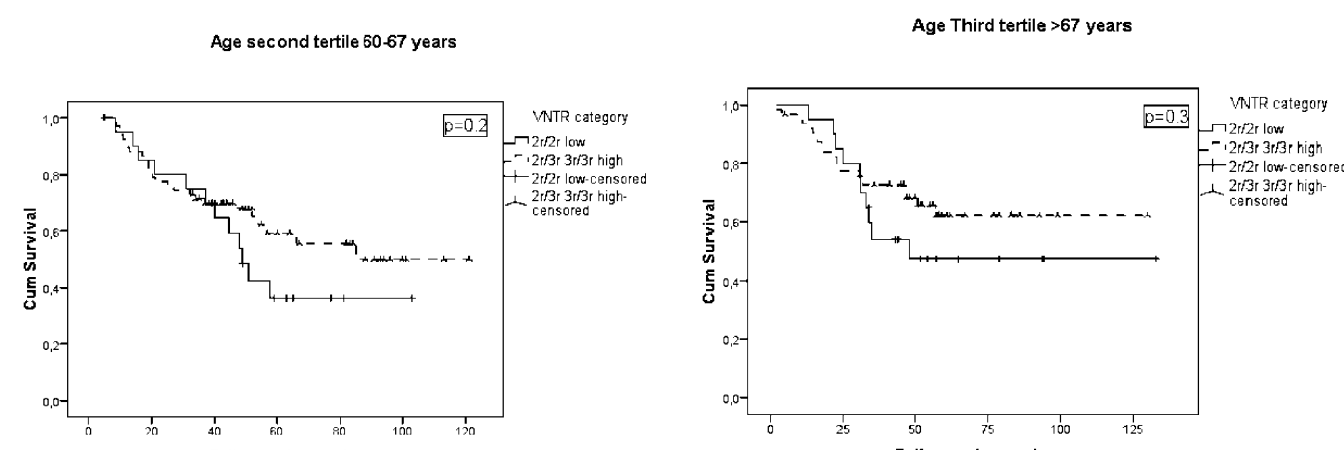

Follow up in months

Fig. 4. Kaplan-Meier curves of the effects on overall survival of the VNTR and SNP categories stratified to age tertiles. (A) SNP category; (B) VNTR category. 
Interestingly, in our population, age seems to play a role on the TS genotype distribution and appears to modify the effects of the genotypes on survival. Indeed, the allelic distribution of both polymorphisms varied depending on age: the median age of the 3RC/3RG genotype was significantly lower in comparison to other genotypes. Similar results have been already reported by Odin et al. The authors described an inverse correlation between TS gene expression and age in colon cancer patients [36]. This relationship could point to a role of the TS gene polymorphisms in colon cancer risk. Hubner et al. described a decreased risk of colon carcinoma between homozygotes for the TS 1491del6 on the $3^{\prime}$-UTR of the gene. However, these authors did not find any role of the polymorphisms on the $5^{\prime}$-UTR [14]. However, further research is needed to study the allelic distribution in the normal population and to see whether this link remains significant.

Furthermore, the effect of the TS genotypes on overall survival was also modified by age. There was a switch in the TS genotypes associated to longer overall survival as age increased. In other words, genotypes associated with low TS expression (homozygous $2 \mathrm{R}$ and the carriers of the $\mathrm{C}$ allele) had a significant positive effect on survival among patients in the first age tertile category (corresponding to patients younger than sixty years). Conversely, these genotypes had a negative effect on survival among patients in the third age tertile category (i.e., older than sixty seven years). In the literature, an inverse association between TS gene expression, and age in colon cancer patients was already described by Odin et al., but the authors did not report its impact on survival.

To elucidate the underlying reasons of this agedependent relation exceeds the scope of this paper and needs additional research. Nevertheless, there is increasing evidence that age affects normal colonic mucosa and tumors. For instance, DNA methylation has been shown to increase with age in normal colonic mucosa $[30,35]$ and different protein expression patterns have been found in the colonic mucosa of the elderly compared to that of younger people [26]. Moreover, Morris et al. have also shown that the molecular aberrations in tumors differ according to age [34]. Thus, our finding supports the hypothesis that age probably modifies the effects of different molecular pathways on oncogenesis and on cancer progression.

In conclusion, the TS polymorphisms in the $5^{\prime}$-UTR are not good markers of 5-FU therapy response in this population of stage III colon carcinoma patients. How- ever, further research is necessary to study the role of age as an effect modifier of the polymorphisms on survival.

\section{Acknowledgements}

We would like to thank, G.J. Creemers MD, O. Repelaer van Driel MD, R.E.H. Smeets MD and A.E.M. Smals MD from the Catharina Hospital, Máxima Medical Centre Eindhoven and the St. Anna Hospital in Geldrop, respectively, for their collaboration in this study.

\section{References}

[1] F. Amatori et al., Thymidylate synthase, dihydropyrimidine dehydrogenase and thymidine phosphorylase expression in colorectal cancer and normal mucosa in patients, Pharmacogenet. Genomics 16 (2006), 809-816.

[2] A. Artinyan et al., Molecular predictors of lymph node metastasis in colon cancer: increased risk with decreased thymidylate synthase expression, J. Gastrointest. Surg. 9 (2005), 12161221.

[3] O. Belvedere et al., Lack of correlation between immunohistochemical expression of E2F-1, thymidylate synthase expression and clinical response to 5-fluorouracil in advanced colorectal cancer, Ann. Oncol. 15 (2004), 55-58.

[4] J. Chen et al., Polymorphism in the thymidylate synthase promoter enhancer region modifies the risk and survival of colorectal cancer, Cancer Epidemiol. Biomarkers Prevent 12 (2003), 958-962.

[5] H.-J. Cho et al., Thymidylate synthase (TYMS) and dihydropyrimidine dehydrogenase (DPYD) polymorphisms in the Korean population for prediction of 5-fluorouracil associated toxicity, Therap. Drug Monitor 29(2) (2007), 190-196.

[6] M. Ciaparrone et al., Predictive role of thymidylate synthase, dihydropyrimidine dehydrogenase and thymidine phosphorylase expression in colorectal cancer patients receiving adjuvant 5-fluorouracil, Oncology 70 (2006), 366-377.

[7] K. Curtin et al., Thymidylate synthase polymorphisms and colon cancer: Associations with tumor stage, tumor characteristics and survival, Int. J. Cancer 120 (2007), 2226-2232.

[8] D. Edler et al., Thymidylate synthase expression: An independent prognostic factor for local recurrence, distant metastasis, disease-free and overall survival in rectal cancer, Clin. Cancer Res. 6 (2000), 1378-1384.

[9] M.E. Fernandez-Contreras et al., Thymidylate synthase expression pattern, expression level and single nucleotide polymorphism are predictors for disease-free survival in patients of colorectal cancer treated with 5-fluorouracil, Int. J. Oncol. 28(5) (2006), 1303-1310.

[10] M. Frattini et al., Different genetic features associated with colon and rectal carcinogenesis, Clin. Cancer Res. 10 (2004), 4015-4021. 
[11] E. Giovannetti et al., Changes in the status of p53 affect drug sensitivity to thymidylate synthase (TS) inhibitors by altering TS levels, Br. J. Cancer 96 (2007), 769-775.

[12] M.J. Gosens et al., Thymidylate synthase genotyping is more predictive for therapy response than immunohistochemistry in patients with colon cancer, Int. J. Cancer 123(8) (2008), 19411949.

[13] E. Hitre et al., Influence of thymidylate synthase gene polymorphisms on the survival of colorectal cancer patients receiving adjuvant 5-fluorouracil, Pharmacogenet. Genomics 15 (2005), 723-730.

[14] R.A. Hubner et al., Thymidylate synthase polymorphisms, folate and B-vitamin intake, and risk of colorectal adenoma, $\mathrm{Br}$. J. Cancer 97(10) (2007), 1449-1456.

[15] B. Iacopetta et al., A polymorphism in the enhancer region of the thymidylate synthase promoter influences the survival of colorectal cancer patients treated with 5-fluorouracil, $\mathrm{Br}$. $\mathrm{J}$. Cancer 85(6) (2001), 827-830.

[16] W. Ichikawa et al., Combination of dihydropyrimidine dehydrogenase and thymidylate synthase gene expressions in primary tumors as predictive parameters for the efficacy of fluoropyrimidine-based chemotherapy for metastaric colorectal cancer, Clin. Cancer Res. 9 (2003), 786-791.

[17] A. Jakobsen et al., Thymidylate synthase and methyltetrahydrofolate reductase gene polymorphism in normal tissue as predictors of fluorouracil sensitivity, J. Clin. Oncol. 23(7) (2005), 1365-1369.

[18] S.A. Jensen, B. Vainer and J.B. Sørensen, The prognostic significance of thymidylate synthase and dihydropyrimidine dehydrogenase in colorectal cancer of 303 patients adjuvantly treated with 5- Fluorouracil, Int. J. Cancer 120 (2006), 694701.

[19] K. Kawakami et al., Polymorphic tandem repeats in the thymidylate synthase gene is associated with its protein expression in human gastrointestinal cancers, Anticancer Res. 19 (1999), 3249-3252.

[20] K. Kawakami et al., Different lengths of a polymorphic repeat sequence in the thymidylate synthase gene affect translational efficiency but not its gene expression, Clin. Cancer Res. 7 (2001), 4096-4101.

[21] K. Kawakami et al., Functional polymorphism of the thymidylate synthase gene in colorectal cancer accompanied by frequent loss of heterozygosity, Jap. J. Cancer Res. 93 (2002), 1221-1229.

[22] K. Kawakami and Y. Watanabe, Identification and functional analysis of single nucleotide polymorphism in the tandem repeat sequence of thymidylate synthase gene, Cancer Res $\mathbf{6 3}$ (2003), 6004-6007.

[23] M. Kornmann et al., Thymidylate synthase and dihydropyrimidine dehydrogenase mRNA expression levels: predictors for survival in colorectal cancer patients receiving adjuvant 5fluorouracil, Clin. Cancer Res. 9 (2003), 4116-4124.

[24] T. Lecomte et al., Thymidylate synthase gene polymorphism predicts toxicity in colorectal cancer patients receiving 5fluorouracil-based chemotherapy, Clin. Cancer Res. 10 (2004), 5880-5888.

[25] H.-J. Lenz et al., p53 and thymidylate synthase expression in untreated stage II colon cancer: associations with recurrence, survival, and site, Clin. Cancer Res. 4 (1998), 1227-1234.

[26] M. Li et al., Proteomic analysis of the aging-related proteins in human normal colon epithelial tissue, J. Biochem. Mol. Biol. 40(1) (2007), 72-81.

[27] T. Liersch et al., Lymph node status and TS gene expression are prognostic markers in stage II/III rectal cancer after neoadjuvant fluorouracil-based chemoradiotherapy, J. Clin. Oncol. 24(25) (2006), 4062-4068.

[28] D.B. Longley, D.P. Harkin and P.G. Johnston, 5-fluorouracil: mechanisms of action and clinical strategies, Nat. Rev. Cancer 3(5) (2003), 330-338.

[29] G. Lurje et al., Thymidylate synthase haplotype is associated with tumor recurrence in stage II and stage III colon cancer, Pharmacogenet. Genomics 18(2) (2008), 161-168.

[30] M.F. de Maat et al., Assessment of methylation events during colorectal tumor progression by absolute quantitative analysis of methylated alleles, Mol. Cancer Res. 5(5) (2007), 461-71.

[31] M.V. Mandola et al., A novel single nucleotide polymorphism within $5^{\prime}$ tandem repeat polymorphism of the thymidylate synthase gene abolishes USF-1 binding and alters transcriptional activity, Cancer Res. 63 (2003), 3898-2904.

[32] E. Marcuello et al., Single nucleotide polymorphism in the $5^{\prime}$ tandem repeat sequences of thymidylate synthase gene predicts for response to fluorouracil-based chemotherapy in advanced colorectal cancer patients, Int. J. Cancer 112 (2004), 733-737.

[33] N.J. Meropol et al., Thymidine phosphorylase expression is associated with response to capecitabine plus irinotecan in patients with metastatic colorectal cancer, J. Clin. Oncol. 24(25) (2006), 4069-4077

[34] M. Morris, C. Platell and B. Iacopetta, A population-based study of age-related variation in clinicopathological features, molecular. Markers and outcome from colorectal cancer, Anticancer Res. 27(4C) (2007), 2833-2838.

[35] H. Nakagawa et al., Age-related hypermethylation of the $5^{\prime}$ region of MLH1 in normal colonic mucosa is associated with microsatellite-unstable colorectal cancer development, Cancer Res. 61(19) (2001), 6991-6995.

[36] E. Odin et al., Colorectal carcinomas with microsatellite instability display increased thymidylate synthase gene expression levels, Clin. Colorectal. Cancer 6(10) (2007), 720-727.

[37] G.J. Peters et al., Induction of thymidylate synthase as 5fluorouracil resistance mechanism, Biochim. Biophys. Acta 1587 (2002), 194-205.

[38] S. Popat, A. Matakidoue and R.S. Houlston, Thymidylate synthase expression and prognosis in colorectal cancer: a systematic review and meta-analysis, J. Clin. Oncol. 22(3) (2004), 529-536.

[39] S. Popat, R. Wort and R.S. Houlston, Inter-relationship between microsatellite instability, thymidylate synthase expression, and p53 status in colorectal cancer: implications for chemoresistance, BMC Cancer 6 (2006), 150-158.

[40] F. Prall et al., Do thymidylate synthase gene promoter polymorphism and the $\mathrm{C} / \mathrm{G}$ single nucleotide polymorphism predict effectiveness of adjuvant 5-fluorouracil-based chemotherapy in stage III colonic adenocarcinoma?, Oncol. Rep. 18(1) (2007), 203-209.

[41] S.T. Pullarkat et al., Thymidylate synthase gene polymorphism determines response and toxicity of 5-FU chemotherapy, Pharmacogenomics J. 1 (2001), 65-70.

[42] A. Ruzzo et al., Pharmacogenetic profiling in patients with advanced colorectal cancer treated with first-line FOLFOX-4 chemotherapy, J. Clin. Oncol. 25(10) (2007), 1247-1254. 
[43] D. Salonga et al., Colorectal tumors responding to 5fluorouracil have low gene expression levels of dihydropyrimidine dehydrogenase, thymidylate synthase and thymidine phosphorylase, Clin. Cancer Res. 6 (2000), 1322-1327.

[44] Y. Shirota et al., ERCC1 and thymidylate synthase mRNA levels predict survival for colorectal cancer patients receiving combination oxaliplatin and fluorouracil chemotherapy, J. Clin. Oncol. 19(23) (2001), 4298-4304.

[45] J. Stoehlmacher et al., A multivariate analysis of genomic polymorphisms: prediction of clinical outcome to 5-FU/oxaliplatin combination chemotherapy in refractory colorectal cancer, $B r$. J. Cancer 91 (2004), 344-354.

[46] K.W. Suh et al., Thymidylate synthase gene polymorphism as a prognostic factor for colon cancer, J. Gastrointest. Surg. 9(3) (2005), 336-342.

[47] T. Tsuji et al., Polymorphism in the thymidylate synthase promoter enhancer region is not an efficacious maker for tumor sensitivity to 5-fluorouracil-based oral adjuvant chemotherapy in colorectal cancer, Clin. Cancer Res. 9 (2003), 3700-3704.

[48] K. Uchida et al., Loss of heterozygosity at the thymidyalte synthase (TS) locus on chromosome 18 affects tumor response and survival in individuals heterozygous for a 28-bp polymorphism in the TS gene, Clin. Cancer Res. 10 (2004), 433-439.

[49] D. Vallböhmer et al., Molecular factors of 5-fluorouracil metabolism in colorectal cancer: Analysis of primary tumor and lymph node metastasis, Int. J. Oncol. 28 (2006), 527-533.

[50] E. Villafranca et al., Polymorphisms of the repeated sequences in the enhancer region of the thymidylate synthase gene promoter may predict downstaging after preoperative chemoradiation in rectal cancer, J. Clin. Oncol. 19(6) (2001), 1779-1786.

[51] J.L. Westra, et al., Predictive value of thymidylate synthase and dihydropyrimidine dehydrogenase protein expression on survival in adjuvantly treated stage III colon cancer patients, Ann. Oncol. 16 (2005), 1646-1653.

[52] Y. Xi et al., Multi-level gene expression profiles affected by thymidylate synthase and 5-fluorouracil in colon cancer, $B M C$ Genomics 7 (2006).

[53] Y. Yanagisawa et al., Modified irinotecan/5-FU/Leucovorin therapy in advanced colorectal cancer and predicting therapeutic efficacy by expression of tumor-related enzymes, Scan. J. Gastroenterol. 42 (2007), 477-484. 


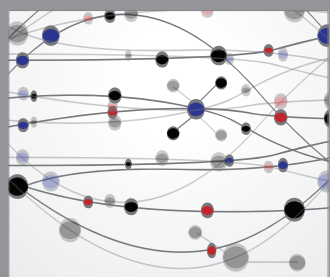

The Scientific World Journal
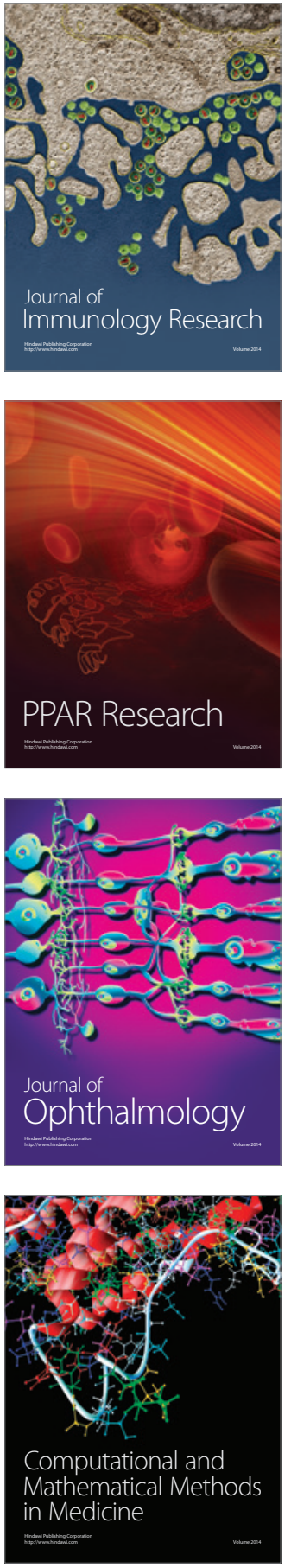

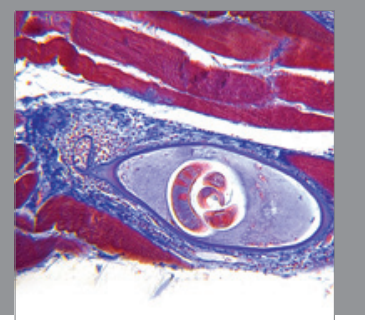

Gastroenterology

Research and Practice
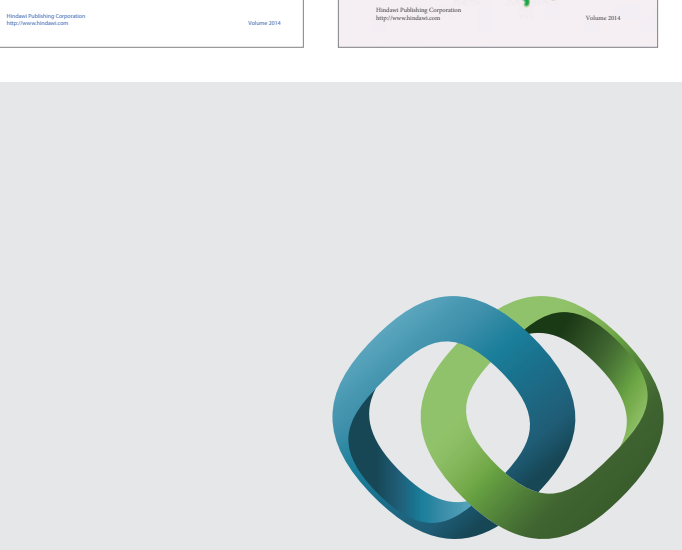

\section{Hindawi}

Submit your manuscripts at

http://www.hindawi.com
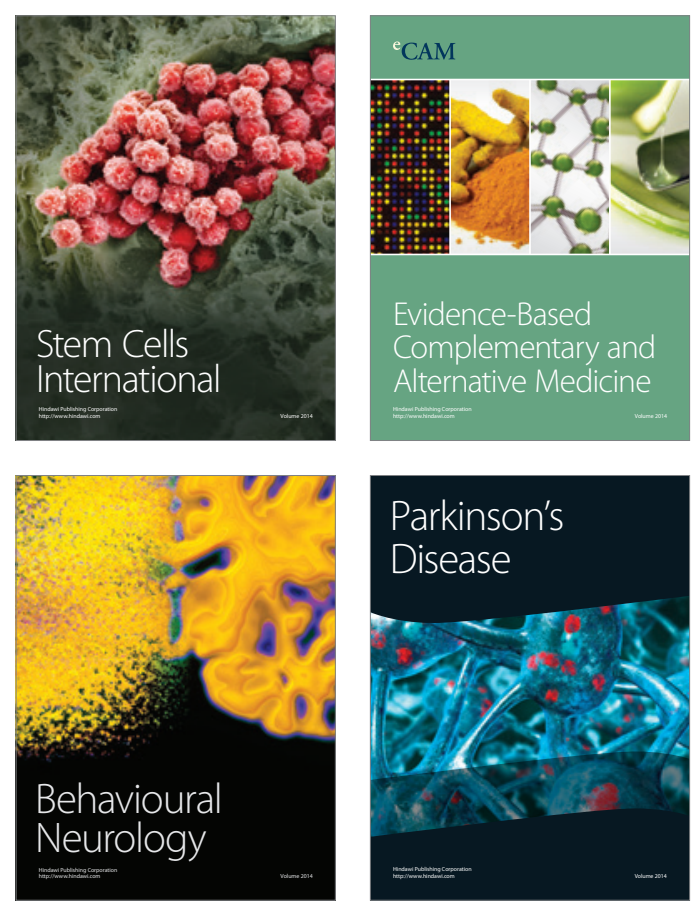

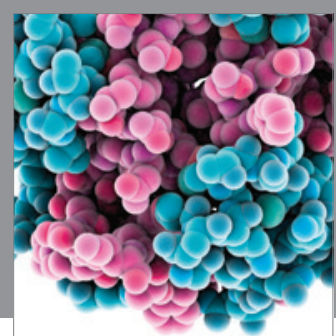

Journal of
Diabetes Research

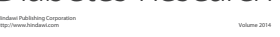

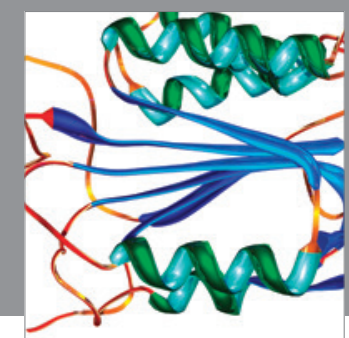

Disease Markers
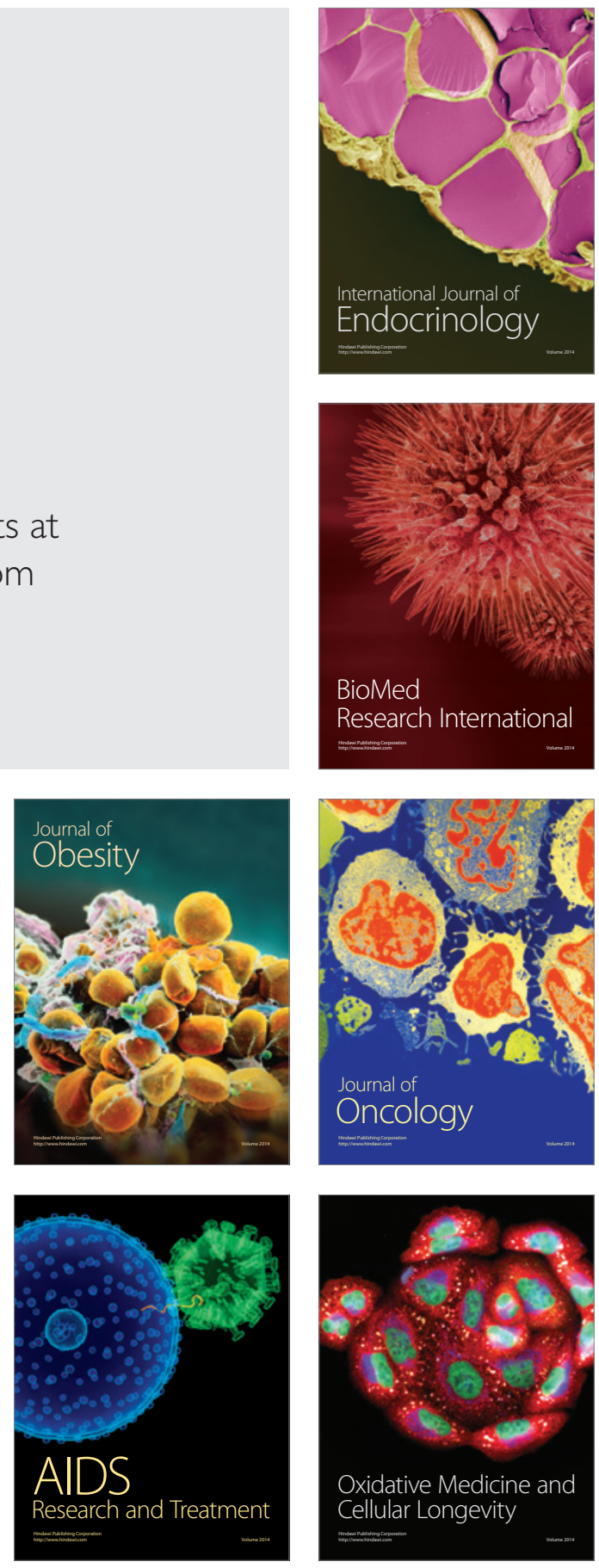